\title{
Diversidad de nematodos marinos de Chile continental y antártico: una evaluación morfológica y molecular
}

\author{
Diversity of marine nematodes from Continental and Antarctic Chile: \\ A morphological and molecular assessment
Natalia Valderrama-Aravena ${ }^{1}$, Karla Pérez-Araneda ${ }^{1}$, Jorge Avaria-Llautureo ${ }^{2}$, Cristián E. Hernández ${ }^{2}$, Matthew Lee $^{3}$ y Antonio Brante ${ }^{1}$

\author{
${ }^{1}$ Departamento de Ecología, Facultad de Ciencias, Universidad Católica de la Santísima Concepción, Alonso de Ribera 2850, \\ Concepción, Chile. abrante@ucsc.cl \\ ${ }^{2}$ Laboratorio de Ecología Evolutiva y Filoinformática, Departamento de Zoología, Facultad de Ciencias Naturales y \\ Oceanográficas, Universidad de Concepción, Alonso de Ribera 2850, Concepción, Chile \\ ${ }^{3}$ Centro i-mar, Universidad de Los Lagos, Camino a Chinquihue km.6, Puerto Montt, Chile
}

\begin{abstract}
Free-living nematodes are an important component of the marine benthos. In Chile there have been few studies on this group, and the majority has focused on the morphological aspect only. In this study, ribosomal 18S RNA and mitochondrial COI genes were used as genetic markers to complement morphological analyses to study the continental and Antarctic Chilean marine nematofauna. Different protocols for fixing, extracting and amplifying DNA were also tested. Not all the possible combinations produced good results. In fact, only the $18 \mathrm{~S}$ gene showed consistently results. Phylogenetic analyses showed some discordance between classical taxonomy (i.e., based on morphology) and molecular data. These results suggest that taxonomic classification using integrative approaches including morphological characteristics and molecular information is needed to study the diversity and evolution of this complex group.
\end{abstract}

Key words: Meiofauna, COI, 18S, nematode

\section{INTRODUCCIÓN}

Los nematodos de vida libre son uno de los grupos de invertebrados más diversos del mundo y componente importante en los ecosistemas bentónicos marinos, desempeñando un papel fundamental en los procesos de descomposición y reciclado de nutrientes (Creer et al. 2010), siendo reconocidos por su potencial como bioindicadores (Bhadury et al. 2008). En sistemas marinos, es uno de los grupos más abundante y diverso dentro de la meiofauna distribuyéndose desde el supralitoral hasta las profundidades abisales del océano (Platt et al. 1984). En Chile, los nematodos marinos han sido escasamente estudiados. Hasta la fecha, se han reconocido un poco más de 300 especies, mayoritariamente de la zona intermareal de la costa continental (Lee et al. 2008).

Una de las principales limitaciones del estudio de los nematodos, es la estimación de su real riqueza y diversidad de especies debido a que su reducido tamaño, alta similitud y complejidad morfológica dificultan su reconocimiento taxonómico (Pereira et al. 2010).

Durante la última década, los marcadores moleculares han sido utilizado como una herramienta complementaria para el estudio de la diversidad intra y interespecífica en diferentes grupos taxonómicos (e.g., Hebert et al. 2003, Hebert \& Gregory 2005, Bucklin et al. 2011). En particular, el código de barras de ADN ha impulsado estudios ecológicos de la biodiversidad en ambientes acuáticos y terrestres (Valentini et al. 2009), así como la investigación sobre la sistemática de organismos marinos utilizando criterios filogenéticos moleculares (Canales-Aguirre et al. 2011). De esta forma, el uso de marcadores moleculares se convierte en una herramienta práctica y eficiente para el estudio de la diversidad y evolución de nematodos marinos, permitiendo implementar una aproximación taxonómica integrada, utilizando información de rasgos morfológicos y moleculares (Bhadury et al. 2006a, b, Grant \& Linse 2009).

El gen ribosomal 18S del ARN y la región de Folmer (Folmer et al. 1994) del gen mitocondrial Citocromo Oxidasa Subunidad I (COI) han sido ampliamente utilizados como códigos de barra genéticos en invertebrados marinos en general (e.g., Blankenship \& Yayanos 2005, Costa et al. 2007). En el grupo de los nematodos de vida libre, ambos 
genes han sido utilizados, aunque los genes ribosomales han presentado mayor éxito (e.g., Bhadury et al. 2006a, Derycke et al. 2010). Sin embargo, los protocolos de fijación, extracción y amplificación del ADN presentan gran variabilidad en su grado de éxito, por lo que ciertas metodologías y análisis deben ajustarse de acuerdo al grupo taxonómico en estudio. En el caso de los nematodos marinos, el reducido tamaño de los individuos dificulta la extracción y éxito de amplificación del ADN. Adicionalmente, los protocolos de extracción de ADN utilizados tradicionalmente conllevan largos tiempos de procesamiento (e.g., Floyd et al. 2002, Bhadury et al. 2006a). Los objetivos del presente trabajo fueron desarrollar un protocolo simple y de reducido tiempo que permita la fijación de las muestras, así como la extracción y amplificación de su ADN; y evaluar el uso de los genes 18S y COI como potenciales marcadores genéticos útiles para el estudio de la diversidad molecular y taxonomía de especies de nematodos marinos de vida libre, provenientes de la costa centro-sur de Chile continental y del intermareal en la Antártica chilena.

\section{MATERIALES Y MÉTODOS}

\section{SITIO DE MUESTREO E IDENTIFICACIÓN MORFOLÓGICA}

Los especímenes de nematodos fueron obtenidos a partir de muestras de sedimento provenientes de la zona intermareal de Iquique (20¹4'S; $\left.70^{\circ} 8^{\prime} \mathrm{O}\right)$, Las Cruces

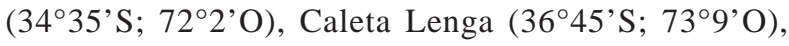
Desembocadura del río Biobío (3648’ S; $73^{\circ} 10^{\circ} \mathrm{O}$ ), Calfuco

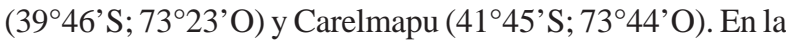
Antártica se muestrearon los sitios de Elefantera (62 $11^{\circ}$ 'S;

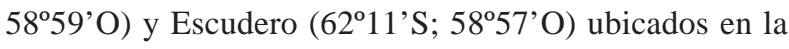
Isla Rey Jorge, Islas Shetland del Sur. Los muestreos se llevaron a cabo entre marzo 2011 y abril 2012. Algunas muestras fueron fijadas en etanol absoluto el cual presenta gran efectividad para conservar el $\mathrm{ADN}$, pero que sin embargo afecta negativamente la morfología de los nematodos, dificultando su posterior caracterización morfológica. De esta forma, un segundo grupo de muestras fueron fijadas en solución DESS (DMSO/EDTA/saturated $\mathrm{NaCl}$ solution; Seutin et al. 1991). El DESS, además de conservar el ADN, en muchos casos ha mostrado éxito en mantener las características morfológicas de los individuos, lo que permite realizar los análisis genéticos sobre los mismos individuos que se realizó la caracterización morfológica y clasificación taxonómica (Yoder et al. 2006). Dado que se utiliza todo el organismo para los análisis genéticos, las muestras fueron previamente transportadas a los laboratorios del Centro i mar, de la Universidad de los Lagos para su clasificación taxonómica mediante caracteres morfológicos y posterior etiquetado. La identificación taxonómica se realizó bajo microscopio (Olympus ${ }^{\circledR}$ BX51 con DIC) según los criterios utilizados por Wieser $(1953,1954,1956)$ y las claves de Platt \& Warwick $(1983,1988)$ and Warwick et al. (1998). En la mayoría de los casos sólo se logró llegar hasta el nivel de género (Tabla 1).

Una vez clasificados morfológicamente, los individuos fueron trasladados a la Facultad de Ciencias de la Universidad Católica de la Santísima Concepción para realizar los análisis genéticos. Los especímenes provenientes de la Desembocadura del río Biobío, dada la cercanía al laboratorio, fueron adicionalmente identificados taxonómicamente y su ADN fue extraído en fresco, para compararlos con las muestras fijadas. Por lo general, se utilizaron 3 individuos por género por cada tratamiento de almacenamiento (etanol absoluto, DESS y en fresco) para ensayar diferentes protocolos de extracción y amplificación en los genes $18 \mathrm{~S}$ y COI. Finalmente, con los protocolos que resultaron amplificaciones positivas, se secuenciaron un total de 53 individuos de las especies más representativas de nematodos continentales y antárticos encontrados en sus respectivas muestras.

\section{EXTRACCIÓN DE ADN}

La extracción de ADN se realizó utilizando 4 protocolos distintos: (1) Modificación al método de Floyd et al. (2002), (2) protocolo de Higuchi (1989), (3) protocolo modificado de Higuchi (1989) y (4) kit comercial de extracción (Kit EZNA ${ }^{\circledR}$ Tissue DNA Omega Biotek $\left.{ }^{\circledR}\right)$. Para el primero, se utilizó $\mathrm{HCl} 1 \mathrm{M}$, Tris-HCl 0.5M (pH 8.0) y Triton ${ }^{\circledR} \mathrm{X}$ 100 e incubación en frío por $8-9 \mathrm{~h} \mathrm{a}-20^{\circ} \mathrm{C}$. Posteriormente se incubaron por $12 \mathrm{~h}$ a $60^{\circ} \mathrm{C}$, calentados por $3 \mathrm{~min}$ a $99^{\circ} \mathrm{C}$ y luego enfriados a temperatura ambiente. Finalmente, los tubos se centrifugaron a 16000 g por $30 \mathrm{~s}$. El protocolo de Higuchi consistió en $100 \mu \mathrm{l}$ de Buffer de Higuchi y $5 \mu \mathrm{l}$ de proteinasa $\mathrm{K}\left(10 \mathrm{mg} \mathrm{ml}^{-1}\right)$ con una incubación de $4 \mathrm{~h}$ a $60^{\circ} \mathrm{C}$, seguido de $15 \mathrm{~min}$ a $94^{\circ} \mathrm{C}$. El protocolo modificado de Higuchi (1989) consistió de $25 \mu$ l de Buffer de Higuchi y $1,25 \mu$ le proteinasa $\mathrm{K}\left(25 \mathrm{mg} \mathrm{ml}^{-1}\right.$ ) incubando por 60 min a $65^{\circ} \mathrm{C}$, seguido de $15 \mathrm{~min}$ a $94^{\circ} \mathrm{C}$. Para la fabricación del buffer de Higuchi se utilizó KCl 0.5M, Tris (pH 8.0) $1 \mathrm{M}, \mathrm{MgCl}_{2}$ 0.5M, Nonidit P40, Tween 20, Gelatin y agua destilada autoclavada. Se incubó por $60 \mathrm{~min}$ a $65^{\circ} \mathrm{C}$ seguido de 15 min a $94^{\circ} \mathrm{C}$ (Denovan et al. 1999). Los protocolos se aplicaron en nematodos almacenados en DESS, etanol absoluto y en fresco (Desembocadura del 
Tabla 1. Clasificación taxonómica al menor nivel posible de los nematodos de vida libre en Chile continental y antártico mediante caracteres morfológicos. Se muestra el número de individuos identificados y secuenciados ( $N$ ), localidades de muestreo y su georeferenciación / Taxonomic classification to the lowest possible rank for free living nematodes from continental Chile and Antarctica using morphological characters. It is showed the number of identified and sequenced individuals (N), sampling sites, and sample GPS coordinates

\begin{tabular}{|c|c|c|c|c|c|c|c|c|}
\hline Familia & Género & Especie & $\mathrm{N}$ & Localidad & $\begin{array}{c}\text { Código } \\
\text { localidad }\end{array}$ & Latitud & Longitud & $\mathrm{N}^{\circ}$ acceso GenBank \\
\hline \multicolumn{9}{|l|}{ Continentales } \\
\hline Axonolaimidae & Odontophora & peritricha & 1 & Las Cruces & Cru & $34^{\circ} 35^{\prime} \mathrm{S}$ & $72^{\circ} 2^{\prime} \mathrm{O}$ & KF591736 \\
\hline Axonolaimidae & Odontophora & peritricha & 1 & Desembocadura & Des & $36^{\circ} 48^{\prime} \mathrm{S}$ & $73^{\circ} 10^{\prime} \mathrm{O}$ & KF591737 \\
\hline Axonolaimidae & Odontophora & peritricha & 1 & Calfuco & Cal & $39^{\circ} 46^{\prime} \mathrm{S}$ & $73^{\circ} 23^{\prime} \mathrm{O}$ & KF591735 \\
\hline Cyatholaimidae & Pomponema & sp. & 1 & Caleta Lenga & Len & $36^{\circ} 45^{\prime} \mathrm{S}$ & $73^{\circ} 9^{\prime} \mathrm{O}$ & KF591743 \\
\hline Cyatholaimidae & Pomponema & sp. & 1 & Desembocadura & Des & $36^{\circ} 48^{\prime} \mathrm{S}$ & $73^{\circ} 10^{\prime} \mathrm{O}$ & KF591742 \\
\hline Enoplidae & Enoplus & sp. & 2 & Desembocadura & Des & $36^{\circ} 48^{\prime} \mathrm{S}$ & $73^{\circ} 10^{\prime} \mathrm{O}$ & KF591719-KF591720 \\
\hline Enoplidae & Enoplus & sp. & 1 & Caleta Lenga & Len & $36^{\circ} 45^{\prime} \mathrm{S}$ & $73^{\circ} 9^{\prime} \mathrm{O}$ & KF591721 \\
\hline Ironidae & Trissonchulus & sp. & 1 & Desembocadura & Des & $36^{\circ} 48^{\prime} \mathrm{S}$ & $73^{\circ} 10^{\prime} \mathrm{O}$ & KF591746 \\
\hline Oncholaimidae & Oncholaimus & sp. & 1 & Desembocadura & Des & $36^{\circ} 48^{\prime} \mathrm{S}$ & $73^{\circ} 10^{\prime} \mathrm{O}$ & KF591739 \\
\hline Oncholaimidae & Oncholaimus & sp. & 1 & Caleta Lenga & Len & $36^{\circ} 45^{\prime} \mathrm{S}$ & $73^{\circ} 9^{\prime} \mathrm{O}$ & KF591741 \\
\hline Selachinematidae & Gammanema & sp. & 1 & Caleta Lenga & Len & $36^{\circ} 45^{\prime} \mathrm{S}$ & $73^{\circ} 9^{\prime} \mathrm{O}$ & KF591723 \\
\hline Thoracostomopsidae & Enoploides & sp. & 10 & Iquique & Iqui & $20^{\circ} 14^{\prime} \mathrm{S}$ & $70^{\circ} 8^{\prime} \mathrm{O}$ & KF591697-KF591706 \\
\hline Thoracostomopsidae & Enoploides & sp. & 3 & Carelmapu & $\mathrm{Cmp}$ & $41^{\circ} 45^{\prime} \mathrm{S}$ & $73^{\circ} 44^{\prime} \mathrm{O}$ & KF591707-KF591708 \\
\hline Thoracostomopsidae & Enoplolaimus & sp. & 1 & Caleta Lenga & Len & $36^{\circ} 45^{\prime} \mathrm{S}$ & $73^{\circ} 9^{\prime} \mathrm{O}$ & KF591718 \\
\hline Thoracostomopsidae & Mesocanthoin & ungulatus & 1 & Caleta Lenga & Len & $36^{\circ} 45^{\prime} \mathrm{S}$ & $73^{\circ} 9^{\prime} \mathrm{O}$ & KF591734 \\
\hline \multicolumn{9}{|l|}{ Antárticos } \\
\hline Chromadoridae & Chromadorita & sp. & 1 & Escudero & Esc & $62^{\circ} 11^{\prime} \mathrm{S}$ & $58^{\circ} 57^{\prime} \mathrm{O}$ & KF591696 \\
\hline Monhysteridae & Halomonhystera & sp. & 2 & Escudero & Esc & $62^{\circ} 11^{\prime} \mathrm{S}$ & $58^{\circ} 57^{\prime} \mathrm{O}$ & KF591724-KF591725 \\
\hline Monhysteridae & Halomonhystera & sp. & 8 & Elefantera & Ele & $62^{\circ} 11$ 's & $58^{\circ} 59^{\prime} \mathrm{O}$ & KF591726-KF591733 \\
\hline Oncholaimidae & -.-.-- & sp. & 1 & Elefantera & Ele & $62^{\circ} 11^{\prime} \mathrm{S}$ & $58^{\circ} 59^{\prime} \mathrm{O}$ & KF591738 \\
\hline Oncholaimidae & Oncholaimus & sp. & 1 & Escudero & Esc & $62^{\circ} 11^{\prime} \mathrm{S}$ & $58^{\circ} 57^{\prime} \mathrm{O}$ & KF591740 \\
\hline Rhabditidae & -..-- & sp. & 2 & Elefantera & Ele & $62^{\circ} 11^{\prime} \mathrm{S}$ & $58^{\circ} 59^{\prime} \mathrm{O}$ & KF591744-KF591745 \\
\hline Selachinematidae & Gammanema & sp. & 1 & Elefantera & Ele & $62^{\circ} 11$ 's & $58^{\circ} 59^{\prime} \mathrm{O}$ & KF591722 \\
\hline Thoracostomopsidae & Enoploides & sp. & 9 & Escudero & Esc & $62^{\circ} 11^{\prime} \mathrm{S}$ & $58^{\circ} 57^{\prime} \mathrm{O}$ & KF591709-KF591717 \\
\hline Tripyloididae & Bathylaimus & sp. & 1 & Escudero & Esc & $62^{\circ} 11^{\prime} \mathrm{S}$ & $58^{\circ} 57^{\prime} \mathrm{O}$ & KF591695 \\
\hline
\end{tabular}

río Biobío). En el caso de los nematodos almacenados en alcohol absoluto, estos fueron lavados previamente con PBS (solución fosfato salina) para remover el alcohol residual y prevenir la inhibición de la PCR.

\section{Marcadores genéticos, AMPLificación POR PCR y SECUENCIACIÓN}

Se ensayaron 2 marcadores genéticos: (1) gen 18S utilizando los partidores MN18F (5'-CGCGAATRGC TCATTACAACAGC-3') y 22R (5’- GCCTGCTGCCTTC CTTGGA-3'; Bhadury et al. 2006a, b); y el gen COI utilizando los partidores universales de Folmer et al. 
(1994), HCO1 (5'-TAAACTTCAGGGTGACCAAAAA ATCA-3') y LCO1 (5'-GGTCAACAAATCATAA AGATATTGG-3'). La reacción en cadena de la polimerasa (PCR) se realizó en un volumen de $50 \mu$ l. Cada solución de PCR contenía: $5 \mu$ l de extracto de ADN con 10X Buffer $\mathrm{MgCl}_{2}$ 2,5 $\mathrm{mM} \mathrm{MgCl}_{2}$, 100X BSA, 2,5 mM dNTPs, $10 \mu \mathrm{M}$

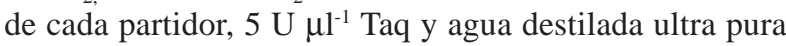
(Invitrogen). Se utilizaron 2 tipos de Taq polimerasa: Taq ADN polimerasa Invitrogen y Toptaq ADN polimerasa Qiagen.

El programa de amplificación para el fragmento seleccionado del gen $18 \mathrm{~S}$ consistió en una desnaturalización inicial a $95^{\circ} \mathrm{C}$ por 5 min, una amplificación de 37 ciclos a $95^{\circ} \mathrm{C}$ por $30 \mathrm{~s}, 56^{\circ} \mathrm{C}$ por $1 \mathrm{~min}, 72^{\circ} \mathrm{C}$ por $1 \mathrm{~min}$ con $30 \mathrm{~s} \mathrm{y}$ finalmente un ciclo de extensión de $72^{\circ} \mathrm{C}$ por 5 min. Para la región de Folmer del gen COI, el programa de amplificación consistió en una desnaturalización inicial de $94^{\circ} \mathrm{C}$ por 1 min, con una amplificación de 35 ciclos a $94^{\circ} \mathrm{C}$ por $30 \mathrm{~s}$, $49^{\circ} \mathrm{C}$ por $55 \mathrm{~s}, 72^{\circ} \mathrm{C}$ por $1 \mathrm{~min}$ con $30 \mathrm{~s}$ y seguido de una extensión final de $72^{\circ} \mathrm{C}$ por $10 \mathrm{~min}$. El producto amplificado se visualizó por electroforesis en gel de agarosa al $2 \%$, teñido con bromuro de etidio $(1 \mu \mathrm{l})$. La purificación y secuenciación de los productos de amplificación fueron realizadas en Macrogen Inc. (Corea del Sur).

\section{EDICIÓN, ALINEAMIENTO Y ANÁLISIS FILOGENÉTICO}

El análisis filogenético se realizó a partir de las secuencias generadas con el marcador $18 \mathrm{~S}$ del ARN debido a la consistencia de los resultados observados para este marcador (ver la sección Resultados y Discusión). Adicionalmente, se utilizaron secuencias del mismo gen de 55 especies de nematodos publicadas en la base de datos moleculares de GenBank para comparar y generar una hipótesis filogenética asociada a las muestras del presente estudio. Se utilizaron como outgroup secuencias de GenBank de las especies Axonchium propinquum (AY284820.1) y Paractinolaimus macrolaimus (AY993978.1). Las secuencias fueron editadas y alineadas en el programa MUSCLE (Edgar 2004) implementado en el programa MEGA 5 (Tamura et al. 2011). Tanto la edición y alineación fueron complementariamente revisadas a ojo para descartar cualquier error de lectura o alineación. Para los análisis filogenéticos, se utilizó el método de Cadenas de Markov Montecarlo (MCMC) dentro de un marco Bayesiano (BMCMC), para estimar la probabilidad $a$ posteriori de los árboles filogenéticos. Debido a que los sitios nucleotídicos de un mismo gen poseen diferentes patrones y tasas de substitución de nucleótidos, se utilizó el modelo mixto general (MM) de Pagel \& Meade (2004, 2008), basado en el modelo General de Tiempo Reversible más tasa de distribución Gama $(\mathrm{GTR}+\Gamma)$ de evolución de secuencias (Rodríguez et al. 1990). El modelo MM acomoda casos en el cual los sitios en el alineamiento evolucionan cualitativamente de diferentes modos, sin la necesidad de un conocimiento a priori de esos patrones, ni partición de los datos. Para seleccionar el número de patrones (o modelos GTR) utilizamos el método MCMC con Salto Reversible (RJMCMC) (Green 1995, Pagel \& Meade 2008). Esta aproximación permite explorar una variedad de posibles modelos y sus parámetros asociados, convergiendo hacia el modelo que se ajusta mejor a los datos en la muestra de árboles. Estos análisis se realizaron en el programa BayesPhylogenies 1.1 ${ }^{1}$. Los análisis BMCMC se llevaron a cabo 4 veces de manera independiente, con 140.000.000 iteraciones de árboles filogenéticos, muestreados cada 10.000 árboles para asegurar que la muestra fuese independiente. Finalmente se seleccionó la cadena que tuvo la mayor verosimilitud marginal. De esta muestra de árboles se excluyeron los primeros 4.000, ya que estos estaban fuera de la zona de convergencia de la Cadena de Markov. Por último, se remuestreó cada 17 árboles para obtener una muestra final de 589 árboles con un bajo valor de autocorrelación $(0,0008)$ en la verosimilitud. Esta muestra final se utilizó para reconstruir el árbol consenso por la regla de la mayoría y estimar los valores de probabilidad a posteriori en el programa BayesTrees 1.3.

Para obtener las relaciones filogenéticas por el criterio de optimización de Máxima Verosimilitud, estimamos el árbol que maximice la verosimilud de nuestras secuencias bajo el modelo GTR $+\Gamma$, utilizando el programa RAxML GUI 1.1 (Silvestro \& Michalak 2010), una interfaz gráfica para RaxML-VI-HPC (Randomized Accelerated Maximmum Likelihod; Stamatakis 2006). Para obtener el soporte de los nodos fue utilizado el método de bootstraping (Felsenstein 1985) con 10.000 pseudoréplicas. Finalmente, para obtener el árbol por el criterio de optimización de Máxima Parsimonia, se utilizó el programa MEGA 5 (Tamura et al. 2011), donde la búsqueda del árbol se realizó mediante la técnica TBR (Tree Bisection and Reconecction). El soporte de los nodos de este árbol se obtuvo mediante bootstraping con 10.000 pseudoréplicas.

${ }^{1}<$ http://www.evolution.reading.ac.uk/BayesPhy.html> 


\section{LIBRERÍA DE CÓDIGOS DE BARRA DE ADN PARA ESPECIES DE NEMATODOS}

La descripción e identificación morfológica de las especies de nematodos analizadas se relacionó con su respectiva secuencia de ADN para el marcador genético, generando una librería tipo códigos de barra de ADN para cada uno de los 53 individuos de nematodos analizados en esta investigación (Tabla 1).

\section{RESULTADOS Y DISCUSIÓN}

Las extracciones de ADN realizadas con la modificación al método de Floyd y la modificación al método de Higuchi presentaron amplificaciones positivas en todas las muestras ensayadas para la región del gen $18 \mathrm{~S}$ y sólo un $10 \%$ para COI (Tabla 2), tanto en aquellas fijadas en etanol absoluto como en DESS (ver abajo). En contraste, el método de Higuchi (no modificado) y el Kit comercial de extracción no mostraron amplificación en estos genes. Si bien el método de Floyd mostró buenos resultados, su protocolo más extenso (aproximadamente 22 h) y demandante de una mayor cantidad de reactivos, lo hacen menos eficiente y más costoso en relación al método de Higuchi modificado que sólo toma 75 min. Este método permite una considerable optimización en tiempo y recursos en comparación a los protocolos comúnmente usados en nematodos (e.g., Bhadury et al. 2006a, b; Bhadury et al. 2008) lo que lo convierte en una excelente y nueva alternativa para realizar estudios genéticos en este grupo. En el caso del método de extracción utilizando el kit comercial, una de las principales limitaciones para su efectividad, es el poco tejido disponible para la extracción, debido a que los nematodos componentes de la meiofauna son usualmente de pequeño tamaño corporal ( $<1$ mm de longitud).

La utilización del marcador $18 \mathrm{~S}$ y el uso de la ADN polimerasa Toptaq ADN de Qiaqen mostraron amplificaciones positivas y consistentes en todas las muestras ensayadas con un tamaño de fragmento de 345 pb (Tabla 2). En contraste, las amplificaciones realizadas con Taq ADN polimerasa de Invitrogen no mostraron resultados positivos en ninguno de los genes, aun cuando posteriormente se ensayó protocolos de amplificación con diferentes concentraciones de $\mathrm{MgCl}_{2}$, partidores y volumen de extracto de ADN. Anteriores estudios en nematodos han mostrado que si bien el gen $18 \mathrm{~S}$ es un gen que muestra moderado polimorfismo (Pereira et al. 2010), presenta alta tasa de éxito en su amplificación, por lo que se ha utilizado como marcador de código de barra genético en este grupo (Floyd et al. 2002, Blaxter 2004, Powers 2004, Bhadury 2006a). Por otra parte, no se obtuvo una amplificación consistente de la región del gen mitocondrial COI con ninguna de la enzimas polimerasas utilizadas, observándose menos del 10\% de muestras amplificadas con Toptaq polimerasa (Qiagen), sin un patrón aparente entre niveles taxonómicos (Tabla 2). Dada su escaso éxito, las pocas muestras amplificadas no fueron secuenciadas. Aunque esta región del gen COI ha sido utilizado con éxito en estudios genéticos en algunas especies de nematodos marinos de vida libre, trabajos anteriores muestran en general que su utilidad pareciera ser especie o grupo específico (e.g., Bhadury et al. 2006a, Derycke et al. 2010).

El método de fijación de los individuos no fue un factor determinante en el éxito de extracción y amplificación, ya que tanto para nematodos en fresco, fijados en DESS como en etanol se obtuvieron los mismos resultados (Tabla 2). Sin embargo, la utilización de DESS tiene la ventaja comparativa de permitir almacenar los ejemplares de nematodos para realizar análisis morfológicos y genéticos sobre los mismos individuos (Yoder et al. 2006).

El estudio morfológico de las muestras reveló un total de al menos 12 géneros distintos pertenecientes a 11 familias (Tabla 1). Al respecto, el análisis filogenético realizado con las secuencias obtenidas en este trabajo y las extraídas desde GenBank para el gen 18S, mostró en términos generales, medios a altos valores de probabilidad a posteriori y de bootstrap. Sin embargo, se observaron inconsistencias para algunos géneros entre la asignación morfológica y la pertenencia a clados filogenéticos. Por ejemplo, los individuos de las localidades antárticas (Ele: Elefantera, Es: Escudero) caracterizados morfológicamente como Halomonhystera sp. y Enoploides sp. se alojaron en clados de otras especies congenéricas o de especies confamiliares encontradas en otras latitudes (Fig. 1). Una situación similar ocurrió en las muestras continentales asignadas a Enoploides sp., en las cuales se observa polifilia. A nivel de familia también se observaron discrepancias entre la clasificación morfológica y la asignación a clados filogenéticos (Fig. 1). Un individuo de la localidad de Lenga clasificado morfológicamente como Gammanema sp., perteneciente a la familia Selachnematidae, correspondería más bien a una especie asociada a la familia Thoracostomopsidae. Además, un individuo de la localidad de la Desembocadura del Biobío clasificado morfológicamente como Pomponema sp. de la familia Cyatholaimidae, pertenecería a una especie de la familia Ironidae. Finalmente, un individuo del sitio 


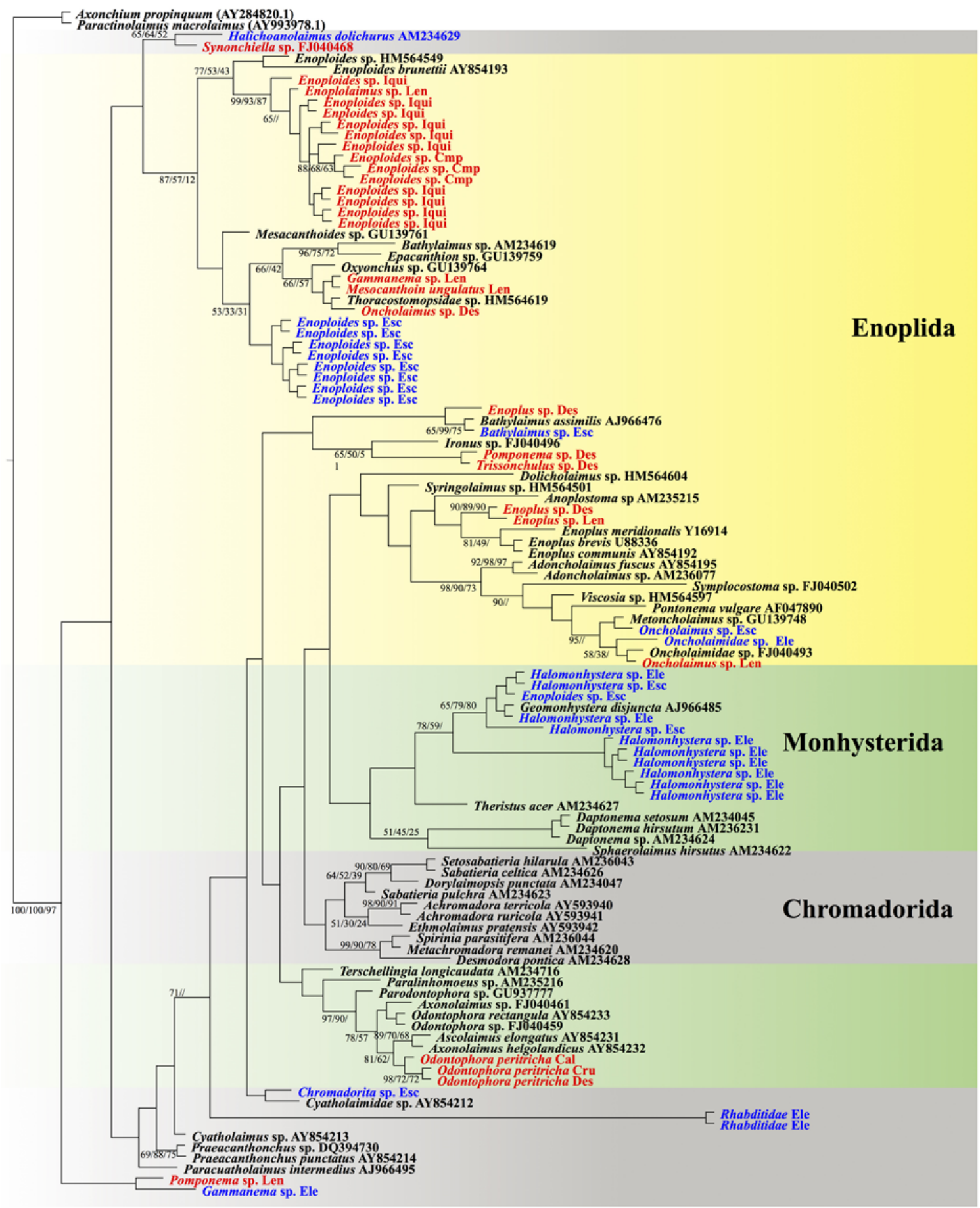

Figura 1. Árbol consenso de la muestra bayesiana obtenido del análisis del gen ribosomal 18S. Para cada individuo se indican las localidades de muestreo, o los códigos de acceso de las secuencias obtenidas de GenBank (Tabla 1). Los nombres específicos en negro indican las secuencias obtenidas de GenBank, en azul las provenientes de la Antártica y en rojo de sitios de Chile continental. Los cuadros en color indican el orden de las especies. Los valores sobre los nodos corresponden a los valores de probabilidad a posteriori (sobre 0,5 ), soporte de bootstrap para ML y MP respectivamente, cuando las topologías fueron similares / Bayesian consensus tree obtained from the analysis of the $18 \mathrm{~S}$ ribosomal gene. For each sequence, sampling location or the GenBank accession number is given (Table 1). Species names in black are from GenBank, in blue from Antarctica, and in green from continental Chile. Nematode Orders are indicated by different color boxes. Values above the nodes indicate the a posteriori probability (> 0.5 only) and bootstrap support for ML and MP analyses, respectively only where the topologies agreed 


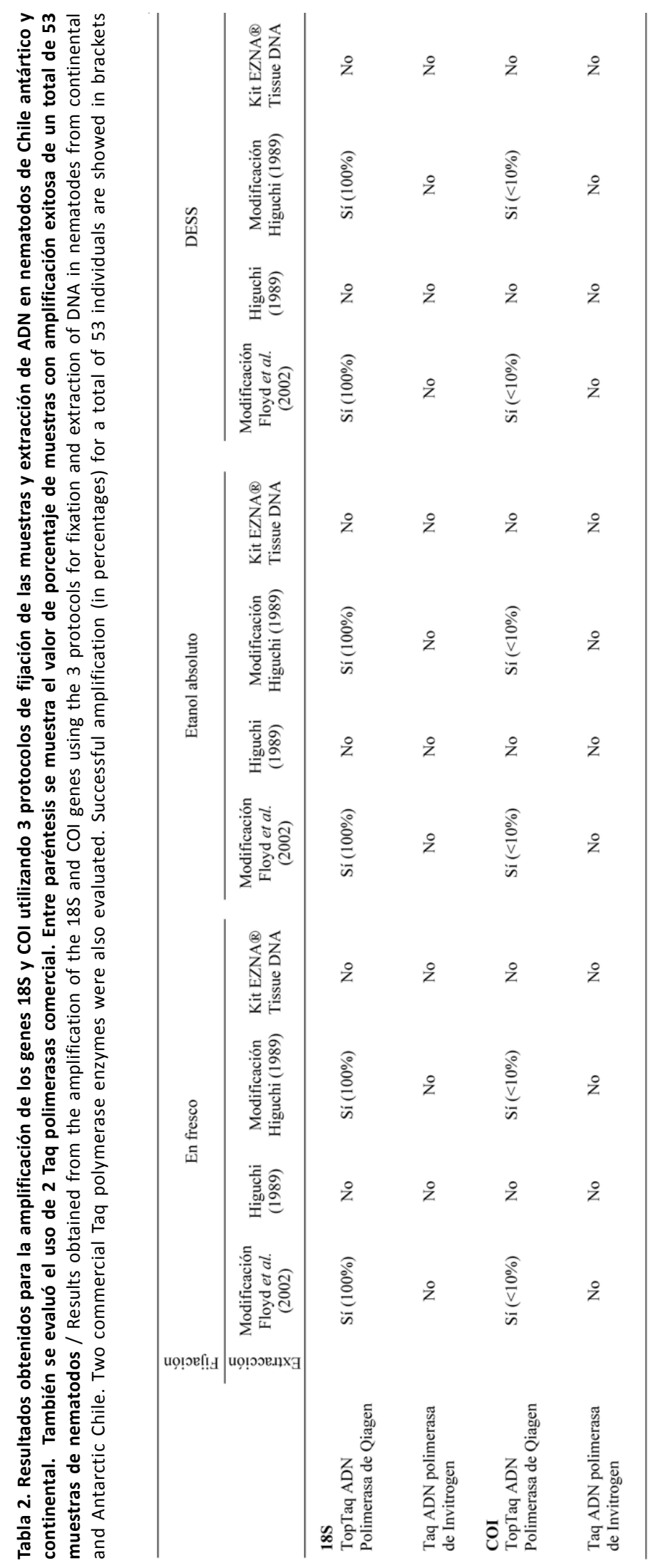

Vol. 49, № 1, 2014 
Antártico Escudero clasificado como Enoploides sp. de la familia Thoracostomopsidae, correspondería a una especie de la familia Xyalidae. De esta forma, a pesar de que el gen $18 \mathrm{~S}$ muestra ser un gen relativamente conservado (Pereira et al. 2010), la discrepancia observada entre la clasificación morfológica y molecular sugiere la potencial existencia de especies crípticas en las costas continentales y antárticas de Chile (Fig. 1). Sin embargo, dado el tamaño del fragmento amplificado (345 pb) y el moderado soporte de algunos nodos, futuros estudios deberían incluir marcadores moleculares adicionales para dar mayor sustento a nuestros resultados. Adicionalmente, es necesario realizar observaciones más minuciosas de los ejemplares que mostraron discrepancias, para poder determinar potenciales diferencias morfológicas o definitivamente considerarlas especies crípticas, y en algunos casos reasignarlas a otros géneros o incluso familias.

Dado que en algunos casos las diferencias morfológicas utilizadas en el presente estudio para la clasificación son significativas como para asumir un error en la identificación, una de las hipótesis que podría explicar algunas inconsistencias a nivel de familia es que se haya secuenciado el contenido estomacal de algunos individuos. Esto se pudo dar especialmente en el individuo Enoploides sp. de la localidad de Escudero, dado que este género es reconocido como un importante depredador de la meiofauna (Galluci et al. 2005). Sin embargo, basados en la potencial existencia de grupos polifiléticos en el análisis filogenético molecular realizado (Fig. 1), apoyamos la hipótesis de diversidad críptica para el grupo de los nematodos, lo que potencia la utilidad de las herramientas moleculares como complemento a la aproximación morfológica clásica para el estudio de su diversidad y evolución. Finalmente, aún queda determinar si las especies encontradas son nuevos registros para Chile o nuevas especies para la ciencia, lo cual es materia actual de investigación.

\section{Agradecimientos}

Agradecemos al Dr. Cristian Gallardo y Valentina Valenzuela del Laboratorio de Genética y Biotecnología Acuícola de la Universidad de Concepción por su colaboración en la estandarización de algunos protocolos de laboratorio. A Marcela Riveros por su ayuda en terreno en la Antártica. El trabajo fue realizado y financiado completamente bajo el marco del proyecto INACh_T_1310. JA-L recibió el apoyo de la beca CONICYT para Magister en Chile.

\section{LITERATURA CITADA}

Bhadury P, M Austen, D Bilton, J Lambshead, A Rogers \& G Smerdon. 2006a. Development and evaluation of a DNA-barcoding approach for the rapid identification of nematodes. Marine Ecology Progress Series 320: 1-9.

Bhadury P, M Austen, D Bilton, J Lambshead, A Rogers \& G Smerdon. 2006b. Molecular detection of marine nematodes from environmental samples: overcoming eukaryotic interference. Aquatic Microbial Ecology 44: 97103.

Bhadury P, M Austen, D Bilton, J Lambshead, A Rogers \& G Smerdon. 2008. Evaluation of combined morphological and molecular techniques for marine nematode (Terschellingia spp.) identification. Marine Biology 154: 509-518.

Blankenship LE \& AA Yayanos. 2005. Universal primers and PCR of gut contents to study marine invertebrate diets. Molecular Ecology 14: 891-899.

Blaxter ML. 2004. The promise of a DNA taxonomy. Philosophical Transactions of the Royal Society B 359: 669-679.

Bucklin A, D Steinke \& L Blanco-Bercial. 2011. DNA Barcoding of marine Metazoa. Annual Review of Marine Science 3: 471-508.

Canales-Aguirre C, N Rozbaczylo \& CE Hernández. 2011. Genetic identification of benthic polychaetes in a biodiversity hotspot in the southeast Pacific. Revista de Biología Marina y Oceanografía 46: 89-94.

Costa FO, JR deWaard, J Boutillier, S Ratnasingham, RT Dooh, M Hajibabaei \& PD Hebert. 2007. Biological identifications through DNA barcodes: the case of the Crustacea. Canadian Journal of Fisheries and Aquatic Sciences 24: 272-295.

Creer S, V Fonseca, D Porazinska, R Giblin-Davis, W Sung, D Power, S Packer, G Carvalho, M Blaxte, P Lambshead \& W Thomas. 2010. Ultrasequencing of the meiofaunal biosphere: practice, pitfalls, and promises. Molecular Ecology 19: 4-20.

Denovan E, J Howlett \& H Robertson. 1999. Simple protocol for extracting nuclear DNA from single embryos of a marine snail. BioTechniques 26: 1050-1052.

Derycke S, P De Ley, I Tandingan De Ley, O Holovachov, A Rigaux \& T Moens. 2010. Linking DNA sequences to morphology: cryptic diversity and population genetic structure in the marine nematode Thoracostoma trachygaster (Nematoda, Leptosomatidae). Zoologica Scripta 39: 276-289.

Edgar R. 2004. MUSCLE: multiple sequence alignment with high accuracy and high throughput. Nucleic Acids Research 32: 1792-1797.

Felsenstein J. 1985. Confidence limits on phylogenies: An approach using the bootstrap. Evolution 39: 783-791. 
Floyd R, A Eyualem, PArtemis \& M Blaxter. 2002. Molecular barcodes for soil nematode identification. Molecular Ecology 11: 839-850.

Folmer O, M Black, W Hoeh, R Lutz \& R Vrijenhoek. 1994. DNA primers for amplification of mitochondrial cytochrome c oxidase subunit I from diverse metazoan invertebrates. Molecular Marine Biology and Biotechnology 3: 294-299.

Grant R \& K Linse. 2009. Barcoding Antarctic Biodiversity: current status and the CAML initiative, a case study of marine invertebrates. Polar Biology 32: 1629-1637.

Green PJ. 1995. Reversible jump Markov chain Monte Carlo computation and Bayesian model determination. Biometrika 82: 711-732.

Hebert P \& R Gregory. 2005. The promise of DNA barcoding for taxonomy. Systematic Biology 54: 852-859.

Hebert P, A Cywinska, S Ball \& J deWaard. 2003. Biological identifications through DNA barcodes. Proceedings of the Royal Society B 270: 313-321.

Higuchi R. 1989. Simple and rapid preparation of samples for PCR. In: Erlich HA (ed). PCR technology principles and applications for DNA amplification, pp. 31-38. Stockton Press, New York.

Lee M, J Castilla, M Fernández, M Clarke, C González, C Hermosilla, L Prado, N Rozbaczylo \& C Valdovinos. 2008. Free-living benthic marine invertebrates in Chile. Revista Chilena de Historia Natural 81: 51-67.

Meade A \& M Pagel. 2008. Modelling heterotachy in phylogenetic inference by reversible-jump Markov chain Monte Carlo. Philosophical Transactions of the Royal Society of London B Biological Sciences 363: 3955-3964.

Pagel M \& A Meade. 2004. A phylogenetic mixture model for detecting pattern-heterogeneity in gene sequence or character-state data. Systematic Biology 53: 571-581.

Platt H, K Shaw \& P Lambshead. 1984. Nematode species abundance patterns and their use in the detection of environmental perturbations. Hydrobiologia 118: 59-66.

Pereira T, G Fonseca, M Mundo-Ocampo, B Guilherme \& A Rocha-Olivares. 2010. Diversity of free-living marine nematodes (Enoplida) from Baja California assessed by integrative taxonomy. Marine Biology 157: 1665-1678.

Platt H \& R Warwick. 1983. Free-living marine nematode. In: Kermack D \& R Barnes (eds). Part I. British enoplids. Synopsis of the British Fauna 28: 1-307. Cambridge University Press, Cambridge.
Platt H \& R Warwick. 1988. Free-living marine nematode. In: Kermack D \& R Barnes (eds). Part II. British chromadorids. Synopsis of the British Fauna 38: 1-502. Cambridge University Press, Cambridge.

Powers T. 2004. Nematode molecular diagnostics: from bands to barcodes. Annual Review of Phytopathology 42: 367-38.

Rodríguez F, J Oliver, A Marín \& R Medina. 1990. The general stochastic model of nucleotide substitution. Journal of Theoretical Biology 142: 485-501.

Seutin G, B White \& P Boag. 1991. Preservation of avian blood tissue samples for DNA analyses. Canadian Journal of Zoology 69: 82-90.

Silvestro D \& I Michalak. 2010. raxmlGUI: A Graphical Front-End for RAxML. <http://sourceforge.net/projects/ raxmlgui/>.

Stamatakis A. 2006. RAxML-VI-HPC: maximum likelihoodbased phylogenetic analyses with thousands of taxa and mixed models. Bioinformatics 22: 2688-2690.

Tamura K, D Peterson, N Peterson, G Stecher, M Nei \& S Kumar. 2011. MEGA5: Molecular evolutionary genetics analysis using maximum likelihood, evolutionary distance, and maximum parsimony methods. Molecular Biology and Evolution 28: 2731-2739.

Valentini A, F Pompanon \& P Taberlet. 2009. DNA barcoding for ecologists. Trends in Ecology and Evolution 24: 110-117.

Warwick R, H Platt \& O Somerfield. 1998. Free-living marine nematode. In: Barnes PR \& J Crothers (eds). Part III. British monhysterids. Synopsis of the British Fauna 53: 1-296. Field Studies Council, Shrewsbury.

Wieser W. 1953. Free-living marine nematodes. I. Enoploidea. Reports of the Lund University Chile Expedition 19481949. 10: 1-155. C.W.K. Gleerup, Lund.

Wieser W. 1954. Free-living marine nematodes. II. Chromadoroidea. Reports of the Lund University Chile Expedition 1948-1949. 17: 1-148. C.W.K. Gleerup, Lund.

Wieser W. 1956. Free-living marine nematodes. III. Axonolaimoidea and Monhysteroidea. Reports of the Lund University Chile Expedition 1948-1949. 26: 1-115. C.W.K. Gleerup, Lund.

Yoder M, I Tandingan De Lay, I King, M Mundo-Ocampo, J Mann, M Blaxter, L Poiras \& P De Ley. 2006. DESS: a versatile solution for preserving morphology and extractable DNA of nematodes. Nematology 8: 367-376. 